Syracuse University

SURFACE

$11-9-2004$

\title{
In Situ Anomalous Small-Angle X-Ray Scattering from Metal Particles in Supported-Metal Catalysts. I. Theory
}

\author{
H. Brumberger \\ Syracuse University \\ D. Hagrman \\ Syracuse University \\ Jerry Goodisman \\ Syracuse University \\ K. D. Finkelstein \\ Cornell University
}

Follow this and additional works at: https://surface.syr.edu/che

Part of the Chemistry Commons

\section{Recommended Citation}

Brumberger, H.; Hagrman, D.; Goodisman, Jerry; and Finkelstein, K. D., "In Situ Anomalous Small-Angle XRay Scattering from Metal Particles in Supported-Metal Catalysts. I. Theory" (2004). Chemistry - Faculty Scholarship. 23.

https://surface.syr.edu/che/23

This Article is brought to you for free and open access by the College of Arts and Sciences at SURFACE. It has been accepted for inclusion in Chemistry - Faculty Scholarship by an authorized administrator of SURFACE. For more information, please contact surface@syr.edu. 
Journal of

\section{Applied Crystallography}

ISSN 0021-8898

Received 28 June 2004

Accepted 9 November 2004

(C) 2005 International Union of Crystallography

Printed in Great Britain - all rights reserved

\section{In situ anomalous small-angle X-ray scattering from metal particles in supported-metal catalysts. I. Theory}

\author{
H. Brumberger, ${ }^{a}+$ D. Hagrman, ${ }^{\text {a J. Goodisman }}{ }^{\mathrm{a} *}$ and K. D. Finkelstein ${ }^{\mathrm{b}}$ \\ ${ }^{a}$ Department of Chemistry, Syracuse University, Syracuse, New York 13244-4100, USA, and \\ ${ }^{b}$ Cornell High Energy Synchrotron Source, Wilson Laboratory, Cornell University, Ithaca, New \\ York 14853, USA. Correspondence e-mail: goodisma@mailbox.syr.edu
}

\begin{abstract}
A supported-metal catalyst can be considered as a mixture of three homogeneous phases: support, void and metal. Information about the metal phase alone can be obtained using anomalous small-angle X-ray scattering (ASAXS), which requires measuring the SAXS for two different wavelengths near the metal's absorption edge. Herein, the conditions that must be obtained so that the difference between the two scattering profiles gives the scattering of the metal alone are presented. In a following contribution, the analysis will be applied to in situ ASAXS measurements made on mordenite impregnated with platinum metal while the temperature and composition of gas in the sample cell are changed. The metal particles are assumed to be randomly distributed spheres with $N(R) \mathrm{d} R$ being the number of spheres with radii between $R$ and $R+\mathrm{d} R$. From $N(R)$ one can obtain the average value of $R$.
\end{abstract}

\section{Introduction}

The activity and properties of a supported-metal catalyst depend, not only on the chemical state of the support and the metal, but also on the interphase surface areas and particle sizes (Wachs, 1992; Stiles \& Koch, 1995). X-rays penetrate a bulk solid, such as a catalyst, non-destructively, making X-ray scattering a possible method for such measurements. Indeed, an early review (Somorjai et al., 1967) characterized smallangle X-ray scattering (SAXS) as 'the most versatile' technique for investigation of the state of the metal in supportedmetal catalysts, and SAXS studies continue to appear ( $\mathrm{Li}$ et al., 2003). Other methods of characterizing supported-metal catalysts include electron microscopy (Jacoby, 2002; Canton et al., 2003), gas adsorption and various spectroscopies; they have been reviewed by Meitzner (1992).

We have previously reported (Brumberger, 1988; Brumberger et al., 1996; Ramaya, 1997) measurement of metal particle sizes in supported-metal catalysts by SAXS. Since $\mathrm{X}$-rays are scattered by the support as well as the metal, we subtracted the scattering of the support alone from the scattering of the catalyst. The validity of subtraction of support scattering from catalyst scattering depends on several assumptions, which may not be completely justified. An alternative means to remove support scattering is by subtracting measurements at two different X-ray energies near the absorption edge of the metal in the catalyst (Naudon, 1995; Creagh, 1999). The atomic scattering factor of the metal is noticeably different for the two energies, whereas the atomic

$\dagger$ Deceased. scattering factors for other elements are the same. Thus, the difference in the scattering of the two wavelengths should consist essentially of the scattering of the metal.

The catalysts we study are zeolites impregnated with platinum. We consider them to consist of three homogeneous phases: zeolite, metal and void. Since surface inhomogeneities are neglected, the X-ray scattering intensity arises from inhomogeneities in the electron density due to phase boundaries. The scattering intensity is proportional to the Fourier transform of the correlation function $\gamma(r)$, which, for a system composed of homogeneous phases, can be written in terms of the 'stick probability functions' $P_{i j}(r) ; P_{i j}(r)$ is the probability that a stick of length $r$, randomly located in the system, has one distinguishable end in phase $i$ and the other in phase $j$ (see below). Consider two three-phase systems with the same $P_{i j}$, but having different electron densities for phase 3. Let their scattering intensities be $I^{\prime}(Q)$ and $I(Q)$. We show in $\S 2$ that the difference, $I^{\prime}(Q)-I(Q)$, has no contribution from the 1-2 interface and that, with a reasonable assumption about $P_{13}$ and $P_{23}, I^{\prime}(Q)-I(Q)$ includes only the scattering from phase 3 .

In our systems, phase 1 is zeolite support, phase 2 is void, and phase 3 is metal. The alteration of the electron density of phase 3 is accomplished by using X-rays with wavelengths near the absorption edge of the metal, and taking advantage of the anomalous scattering (Georgopoulos \& Cohen, 1985; Epperson \& Thiyagarajan, 1988; Creagh, 1999). Near the absorption edge, the index of refraction and hence the scattering power of the metal vary noticeably with wavelength, so measuring the scattering at two different wavelengths is equivalent to measuring the scattering from two systems with 
different values of $n_{3}$, the electron density of phase 3 . The source of monochromatic X-rays of appropriate wavelengths is the Cornell High Energy Synchrotron Source (CHESS). An additional advantage of synchrotron radiation is the much higher intensity, which makes it possible to obtain the X-ray scattering profile $I(Q)$ for each wavelength in seconds rather than many minutes. Thus $I(Q)$ can be obtained for a system in which the correlation function or the particle size distribution changes with time.

The use of anomalous small-angle X-ray scattering for problems in materials science has recently been reviewed by Goerigk et al. (2003). ASAXS has now been used by a number of workers to study the size distribution of catalyst particles in porous supports, as we do in the present work (Haubold et al., 1996, 1997, 1999; Benedetti et al., 1999; Rasmussen et al., 2000; Bóta et al., 2002; Bönnemann et al., 2002; Vad et al., 2002; Polizzi et al., 2002; Canton et al., 2003; Haubold, 2003).

\section{SAXS and anomalous scattering}

For an isotropic system, the scattering intensity at an angle $\theta$ is given by (Brumberger, 1968; Goodisman \& Brumberger, 1971; Schmidt, 1995)

$$
I(Q)=4 \pi V\left\langle\eta^{2}\right\rangle I_{\mathrm{e}}(Q) \int_{0}^{\infty} r^{2} \gamma(r) \frac{\sin (Q r)}{Q r} \mathrm{~d} r .
$$

Here, $Q=4 \pi \lambda^{-1} \sin (\theta / 2)$ with $\lambda$ the wavelength of the radiation and $\theta$ the scattering angle; $V$ is the sample volume, $\gamma$ is the density-density correlation function, and $\left\langle\eta^{2}\right\rangle$ is the meansquare electron density fluctuation from the mean. $I_{\mathrm{e}}(Q)$ is the Thomson scattering of a single electron, which is essentially independent of $\theta$ for small scattering angles. If the system is composed of several homogeneous phases, $i$, each of uniform electron density $n_{i}$,

$$
\left\langle\eta^{2}\right\rangle=\sum_{i} \varphi_{i}\left(n_{i}-\langle n\rangle\right)^{2},
$$

where $\varphi_{j}$ is the volume fraction of phase $j$, and $\langle n\rangle$ the average electron density, $\sum_{i} \varphi_{i} n_{i}$. In principle, the correlation function can be determined from the Fourier transform of the scattering intensity $I(Q)$ (Schmidt, 1995).

The correlation function for a system of homogeneous phases with sharp boundaries may be written as

$$
\gamma(r)=\frac{1}{\left\langle\eta^{2}\right\rangle}\left[\sum_{i, j} P_{i j}(r) n_{i} n_{j}-\langle n\rangle^{2}\right],
$$

where $P_{i j}(r)$ is the 'stick probability function': the probability that a stick of length $r$, located at random in the system, has one end in phase $i$ and the other in phase $j$ (Goodisman \& Brumberger, 1971; Ramaya, 1997). There are three stick probability functions $P_{i j}$ for a three-phase system such as a supported-metal catalyst. In the catalyst, $i=1,2$ or 3 refers to support, void or metal, respectively. It is obvious that $P_{i j}(0)=$ $\varphi_{j} \delta_{i j}$, so that $\gamma(0)=1$. It can also be shown (Goodisman \& Brumberger, 1971) that $\left(\mathrm{d} P_{i j} / \mathrm{d} r\right)_{r=0}=S_{i j} / 4 V$, where $S_{i j}$ is the interphase surface area; thus the correlation function contains information about the interphase surface areas. It is clear, however, that one cannot find an individual surface area from $\gamma(r)$ without additional assumptions.

For X-ray wavelengths near the absorption edge of one phase, the scattering power, equivalent to the electron density, of that phase varies noticeably with wavelength (anomalous scattering). Carrying out SAXS measurements at two wavelengths near the absorption edge is equivalent to performing SAXS measurements on two systems having the same structure (volume fractions, interphase surface areas, etc.) but different electron densities for one phase (phase 3, the metal, in our systems). If the electron densities for phase 3 are $n_{3}$ and $n_{3}^{\prime}$ in the two systems, the difference in the SAXS for the two wavelengths is

$$
\begin{aligned}
I(Q)-I^{\prime}(Q)= & 4 \pi V I_{\mathrm{e}}(Q) \int_{0}^{\infty} \mathrm{d} r r^{2} \frac{\sin (Q r)}{Q r} \\
& \times\left[2\left(P_{13} n_{1}+P_{23} n_{2}\right)\left(n_{3}-n_{3}^{\prime}\right)\right. \\
& -2\left(\varphi_{1} n_{1}+\varphi_{2} n_{2}\right) \varphi_{3}\left(n_{3}-n_{3}^{\prime}\right) \\
& \left.+P_{33}\left(n_{3}^{2}-n_{3}^{\prime 2}\right)-\left(\varphi_{3} n_{3}\right)^{2}+\left(\varphi_{3} n_{3}^{\prime 2}\right)\right],
\end{aligned}
$$

which has no contribution from $P_{12}$. The bracketed part of this equation is equal to

$$
\begin{aligned}
& \left(n_{3}-n_{3}^{\prime}\right)\left[2 P_{13} n_{1}+2 P_{23} n_{2}-2 \varphi_{3}\left(\varphi_{1} n_{1}+\varphi_{2} n_{2}\right)\right. \\
& \left.\quad+\left(P_{33}-\varphi_{3}^{2}\right)\left(n_{3}+n_{3}^{\prime}\right)\right]
\end{aligned}
$$

so that

$$
\begin{aligned}
I(Q)-I^{\prime}(Q)= & 8 \pi V I_{\mathrm{e}}(Q)\left(n_{3}-n_{3}^{\prime}\right) \\
& \times \int_{0}^{\infty} \mathrm{d} r r^{2} \frac{\sin (Q r)}{Q r}\left[P_{13} n_{1}+P_{23} n_{2}+P_{33} \bar{n}_{3}\right. \\
& \left.-\varphi_{3}\left(\varphi_{1} n_{1}+\varphi_{2} n_{2}+\varphi_{3} \bar{n}_{3}\right)\right]
\end{aligned}
$$

where $\bar{n}_{3}$ is the average of $n_{3}$ and $n_{3}^{\prime}$. We now show under what circumstances $I(Q)-I^{\prime}(Q)$ is equal to the scattering of a twophase system.

The phases 1 and 2 together may be considered a single phase $A$, with volume fraction $\varphi_{A}=\varphi_{1}+\varphi_{2}$ and average electron density

$$
n_{A}=\frac{\varphi_{1} n_{1}+\varphi_{2} n_{2}}{\varphi_{1}+\varphi_{2}}
$$

Phase 3 is now denoted as $B$, with volume fraction $\varphi_{B}=\varphi_{3}$ and electron density $n_{B}=\bar{n}_{3}$. The stick probability $P_{B B}$ is $P_{33}$ and $P_{A A}$ is $P_{11}+P_{12}+P_{21}+P_{22}$. It is assumed that

$$
\frac{P_{13}}{P_{A B}}=\frac{\varphi_{1}}{\varphi_{A}}=\frac{\varphi_{1}}{\varphi_{1}+\varphi_{2}}
$$

and similarly for $P_{23}$, i.e. that phases 1 and 2 are arranged randomly in the new combined phase $A$. Then equation (5) may be written as 


$$
\begin{aligned}
I(Q)-I^{\prime}(Q)= & 8 \pi V I_{\mathrm{e}}(Q)\left(n_{3}-n_{3}^{\prime}\right) \\
& \times \int_{0}^{\infty} \mathrm{d} r r^{2} \frac{\sin (Q r)}{Q r}\left[n_{A}\left(P_{A B}-\varphi_{A} \varphi_{B}\right)\right. \\
& \left.+n_{B}\left(P_{B B}-\varphi_{B} \varphi_{B}\right)\right] .
\end{aligned}
$$

For a two-phase system with phases $a$ and $b$, the scattering intensity is given by

$$
\begin{aligned}
I_{2}(Q)= & 4 \pi V I_{\mathrm{e}}(Q) \int_{0}^{\infty} \mathrm{d} r r^{2} \frac{\sin (Q r)}{Q r}\left[n_{a}^{2}\left(P_{a a}-\varphi_{a}^{2}\right)\right. \\
& \left.+2 n_{a} n_{b}\left(P_{a b}-\varphi_{a} \varphi_{b}\right)+n_{b}^{2}\left(P_{b b}-\varphi_{b}^{2}\right)\right]
\end{aligned}
$$

Since $P_{a b}+P_{b b}=\varphi_{b}$ and $P_{a b}+P_{a a}=\varphi_{a}$, this may be rearranged to

$$
\begin{aligned}
I_{2}(Q)= & 4 \pi V I_{\mathrm{e}}(Q) \int_{0}^{\infty} \mathrm{d} r r^{2} \frac{\sin (Q r)}{Q r}\left[\left(P_{a b}-\varphi_{a} \varphi_{b}\right)\left(n_{a} n_{b}-n_{a}^{2}\right)\right. \\
& \left.+\left(P_{b b}-\varphi_{a}^{2}\right)\left(n_{b}^{2}-n_{a} n_{b}\right)\right] \\
= & 4 \pi V I_{\mathrm{e}}(Q)\left(n_{b}-n_{a}\right) \int_{0}^{\infty} \mathrm{d} r r^{2} \frac{\sin (Q r)}{Q r}\left[\left(P_{a b}-\varphi_{a} \varphi_{b}\right) n_{a}\right. \\
& \left.+\left(P_{b b}-\varphi_{b}^{2}\right) n_{b}\right] .
\end{aligned}
$$

This is identical to equation (6) if $\left(n_{b}-n_{a}\right)$ is replaced by $2\left(n_{3}^{\prime}-n_{3}\right)$. Thus our assumption of randomness, $P_{13} / P_{A B}=$ $\varphi_{1} / \varphi_{A}$, makes the difference in scattering intensities for the two systems equal to the scattering intensity from a two-phase system. One of the phases is the metal and the other a densityaveraged support. The effective electron density difference is $2\left(n_{3}^{\prime}-n_{3}\right)$ rather than the difference in electron densities of metal and support.

The electron density difference $\left(n_{3}^{\prime}-n_{3}\right)$ is actually the difference in atomic scattering factors for two different X-ray wavelengths. In general, the coherent atomic scattering factor $f(Q, E)$ for X-rays of energy $E(E=h c / \lambda)$ is given by (Haubold et al., 1994; Naudon, 1995; Cross et al., 1998)

$$
f(Q, E)=f_{\mathrm{o}}(Q)+f^{\prime}(Q, E)+i f^{\prime \prime}(Q, E),
$$

where $f_{\mathrm{o}}$, independent of $E$, is the Fourier transform of the electron density. In the small-angle scattering range, the $Q$ dependence, or dependence on scattering angle, can be neglected, and $f_{\mathrm{o}}$ becomes equal to the atomic number $Z$. The term $f^{\prime \prime}$ is proportional to the absorption coefficient, and $f^{\prime}$ is related to $f^{\prime \prime}$ through a Kramers-Krönig relation,

$$
f^{\prime}(\omega)=\frac{2}{\pi} \int_{0}^{\infty} \frac{\omega^{\prime} f^{\prime \prime}\left(\omega^{\prime}\right)}{\omega^{\prime 2}-\omega^{2}} \mathrm{~d} \omega^{\prime}
$$

where $\omega=2 \pi c / \lambda$. The absorption coefficient $f^{\prime \prime}$ is small for low $\omega$, but becomes large when $\omega$ approaches an absorption frequency. Then $f^{\prime}(\omega)$ becomes large and negative near the absorption edge, varying rapidly with $\omega$, making the real part of the scattering factor, $f_{\mathrm{o}}+f^{\prime}(E)$, vary markedly over a small range of $E$. Thus the electron number $Z$ is reduced by several electrons or more within $10 \mathrm{eV}$ from the absorption edge (Naudon, 1995; Goerigk et al., 2003), and the effective electron density of one phase in a multi-phase system decreases as the $\mathrm{X}$-ray frequency approaches the absorption frequency.

Therefore the difference $I(Q)-I^{\prime}(Q)$ for a three-phase system, where X-rays of two different frequencies are used to obtain $I^{\prime}(Q)$ and $I(Q)$, has the form of the X-ray scattering from a two-phase system [equations (6) and (7)]. One of the phases is that with a scattering cross section which is very different for the two frequencies (the metal in the present application), and the other an average of the other phases (zeolite and void). Instead of being proportional to the electron density difference between the two phases, the scattering intensity is proportional to the difference in effective electron densities of the metal at the two wavelengths used. When this theory is applied to the scattering of a supported-metal catalyst (Brumberger et al., 2005), only the variation of scattering intensity with $Q$ is important, and not the value of the proportionality constant.

\section{3. $\boldsymbol{P}_{i j}$ for a system of particles}

The metal (phase 3 ) is expected to be in the form of particles, embedded in the mixed zeolite and void phases. We must now calculate the relevant $P_{i j}$ for this situation. The particles are assumed to be all of the same shape but of different sizes, with the probability that a particle chosen at random has characteristic radius $R$ being

$$
N(R)=\exp (-\alpha R)\left(\beta+\gamma R+\delta R^{2}\right),
$$

where $\alpha, \beta, \gamma$ and $\delta$ are parameters. Normalization requires

$$
\int_{0}^{\infty} N(R) \mathrm{d} R=1
$$

Let $A(r ; R)$ be the autocorrelation function for a particle of size $R$, i.e. the probability (averaged over angles) that, if a stick of length $r$ has one end in a particle of size $R$, the other end is within the same particle. Obviously, $A(0 ; R)=1$ and $A(r ; R) \rightarrow 0$ as $r \rightarrow \infty$.

The stick probability function $P_{33}$ starts with $\varphi_{3} N(R)$ : the probability that one end of the stick lies in a particle of size $R$ of phase 3. This is multiplied by a sum of two terms, representing the probability that the other end lies in the same particle or in another particle of phase 3 , and then integrated over $R$.

$$
P_{33}(r)=\varphi_{3} \int_{0}^{\infty} N(R)\left\{A(r ; R)+[1-A(r ; R)] \varphi_{3}\right\} \mathrm{d} R .
$$

In addition, we have

$$
P_{3 j}(r)=\varphi_{3} \int_{0}^{\infty} N(R)[1-A(r ; R)] \varphi_{j} \mathrm{~d} R,
$$

where $j=1$ or 2 . Note that $P_{13} / P_{23}=\varphi_{1} / \varphi_{2}$ as required by our model. The stick probability $P_{12}$ need not be calculated since it 
cancels out in $I^{\prime}(Q)-I(Q)$. Substituting the stick probabilities into equation (6), we find

$$
\begin{aligned}
{[I(Q)} & \left.-I^{\prime}(Q)\right] / 8 \pi V I_{\mathrm{e}}(Q)\left(n_{3}-n_{3}^{\prime}\right) \\
= & \int_{0}^{\infty} \mathrm{d} r r^{2} \frac{\sin (Q r)}{Q r}\left\{n_{A}\left[P_{13}+P_{23}-\left(1-\varphi_{3}\right) \varphi_{3}\right]\right. \\
& \left.+\bar{n}_{3}\left(P_{33}-\varphi_{3} \varphi_{3}\right)\right\} \\
= & \int_{0}^{\infty} \mathrm{d} r r^{2} \frac{\sin (Q r)}{Q r}\left[-n_{A}\left(1-\varphi_{3}\right) \varphi_{3} \int_{0}^{\infty} N(R) A(r ; R) \mathrm{d} R\right. \\
& \left.+\bar{n}_{3} \varphi_{3} \int_{0}^{\infty} N(R) A(r ; R) \mathrm{d} R\left(1-\varphi_{3}\right)\right]
\end{aligned}
$$

with $n_{A}\left(\varphi_{1}+\varphi_{2}\right)=n_{1} \varphi_{1}+n_{2} \varphi_{2}$. This further simplifies to

$$
\begin{aligned}
\frac{I(Q)-I^{\prime}(Q)}{8 \pi V I_{\mathrm{e}}(Q)\left(n_{3}-n_{3}^{\prime}\right)}= & \left(\bar{n}_{3}-n_{A}\right) \varphi_{3}\left(1-\varphi_{3}\right) \\
& \times \int_{0}^{\infty} N(R)\left[\int_{0}^{\infty} \mathrm{d} r r^{2} \frac{\sin (Q r)}{Q r} A(r ; R)\right] \mathrm{d} R .
\end{aligned}
$$

The Fourier transform of $A(r ; R)$ gives $I^{\mathrm{sph}}(Q)$, the scattering of a sphere of radius $R$. Thus the scattering is the sum of contributions of the individual particles (Schmidt, 1995).

For a sphere with uniform electron density $\rho$, the scattering intensity is given by (Glatter, 1995)

$$
I^{\mathrm{sph}}(Q)=(4 \pi \rho)^{2}[\sin (Q a)-Q a \cos (Q a)]^{2} / Q^{6} .
$$

Equation (13) may be approximated by a non-oscillating Gaussian function (Somorjai et al., 1967; Hukins, 1981); in fact a Gaussian may be used to approximate the scattering intensity of particles of any shape (Guinier approximation):

$$
I^{\text {part }}(Q)=I(0) \exp \left(-Q^{2} G^{2} / 3\right)
$$

Here $G$ is the radius of gyration and $I(0)$ is equal to the electron density squared multiplied by the square of the particle volume (Hukins, 1981; Schmidt, 1995). For a sphere of radius $a, G^{2}=\frac{3}{5} a^{2}$.

In previous work (Brumberger et al., 1996), we assumed the metal was in the form of independent particles with variable radii of gyration. We used the Guinier approximation and defined $F(G) \mathrm{d} G$ as the number of particles with radius of gyration between $G$ and $G+\mathrm{d} G$. The total scattering intensity is proportional to

$$
I^{\mathrm{tot}}(Q)=\int_{0}^{\infty} \mathrm{d} G F(G) G^{6} \exp \left(-G^{2} Q^{2} / 3\right) .
$$

However, this does not lead to Porod behavior (intensity proportional to $Q^{-4}$ for $Q \rightarrow \infty$ ), expected for particle scattering (Schmidt, 1995; Schaefer et al., 1995). The reason is that the Guinier approximation is appropriate for small $Q$ but not for $Q \rightarrow \infty$.
The complete sphere scattering function equation (13) does give Porod behavior when averaged over the sinusoidal oscillations. Therefore, we represent the metal particles as a collection of spheres of different sizes and use equation (13). With equation (9) for $N(R)$ we have the total difference scattering proportional to

$$
\begin{aligned}
I_{\mathrm{t}}(Q)= & \int_{0}^{\infty} \mathrm{d} R \exp (-\alpha R)\left(\beta+\gamma R+\delta R^{2}\right)(4 \pi \rho)^{2} \\
& \times\left\{[\sin (Q R)-Q R \cos (Q R)]^{2} / Q^{6}\right\} .
\end{aligned}
$$

The integral can be evaluated in closed form.

The values of the four parameters $(\alpha, \beta, \gamma, \delta)$ in $N(R)$ are chosen to obtain the best fit of $I_{\mathrm{t}}(Q)$ to the experimental $I(Q)$. Specifically, we minimize the sum of the relative deviations,

$$
S=\sum_{j}\left[\frac{I\left(Q_{j}\right)-I_{\mathrm{t}}\left(Q_{j}\right)}{I\left(Q_{j}\right)}\right]^{2}
$$

with respect to all four parameters. The sum in equation (15) is over the values of $Q$ for which scattering intensity can be measured reliably. With the values of the parameters determined, we calculate properties of the distribution.

\section{Discussion and conclusions}

In this and a following paper, we analyze the SAXS from a supported-metal catalyst, modeled as a system of three homogeneous phases with sharp phase boundaries. We first show [equations (2)-(7)] that, for such a system, the difference in the SAXS at two X-ray wavelengths is equivalent to the scattering of a two-phase system. If the two wavelengths are near the absorption edge for one of the phases (phase 3), the effect of changing wavelength is to change the effective scattering power or electron density of phase 3 , while leaving the distribution of phases the same. In the two-phase system, one phase is phase 3 of the three-phase system (the metal in the present example) and the other phase is an average of phases 1 and 2 of the three-phase system.

If the metal is modeled as a collection of spheres of variable radii, the difference scattering is shown [equations (10)-(12)] to be proportional to the sum of scattering intensities from the spheres. The sphere scattering function [equation (13)] is multiplied by the radius distribution function [equation (9)] and integrated over $R$ to give the theoretical scattering intensity curve $I_{\mathrm{t}}$ [equation (14)]. The correct sphere scattering function [equation (9)] was used in preference to the Guinier approximation because the latter does not give the correct Porod-law behavior, $I Q^{4} \rightarrow$ constant as $Q \rightarrow \infty$.

This work is based upon research conducted at the Cornell High Energy Synchrotron Source, which is supported by the National Science Foundation and the National Institutes of Health/National Institute of General Medical Sciences under award DMR 9713424. 


\section{References}

Benedetti, A., Bertoldo L., Canton, P., Goerigk, G., Pinna, F., Riello, P. \& Polizzi, S. (1999). Catal. Today, 49, 485-489.

Bönnemann, H., Waldoefner, N., Haubold, H.-G. \& Vad, T. (2002). Chem. Mater. 14, 1115-1120.

Bóta, A., Goerigk, G., Drucker, T., Haubold, H.-G. \& Petro, J. (2002). J. Catal. 205, 354-357.

Brumberger, H. (1968). X-ray and Electron Methods of Analysis, edited by H. van Olphen \& W. Parrish, ch. IV. New York: Plenum.

Brumberger, H. (1988). Makromol. Chem. Macromol. Symp. 15(Discuss. Conf.), 223-230.

Brumberger, H., Goodisman, J., Ramaya, R. \& Ciccariello, S. (1996). J. Appl. Cryst. 29, 526-530.

Brumberger, H., Hagrman, D. \& Goodisman, J. (2005). J. Appl. Cryst. Submitted.

Canton, P., Menegazzo, F., Polizzi, S., Pinna, F., Pernicone, N., Riello, P., Fagherazzi, G. \& Consorzio INSTM UdR Venezia (2003). Catal. Lett. 88, 141-146.

Creagh, D. C. (1999). International Tables for Crystallography, Vol. C, 2nd ed., edited by A. J. C. Wilson \& E. Prince, Section 4.2.6. Dordrecht: Kluwer.

Cross, J. O., Newville, M., Rehr, J. J., Sorensen, L. B., Bouldin, C. E., Watson, G., Gouder, T., Lander, G. H. \& Bell, M. I. (1998). Phys. Rev. B, 58, 11215-11225.

Epperson, J. E. \& Thiyagarajan, P. (1988). J. Appl. Cryst. 21, 652662.

Georgopoulos, P. \& Cohen, J. B. (1985). J. Catal. 92, 211-215.

Glatter, O. (1995). Modern Methods of Data Analysis in Small-Angle Scattering and Light Scattering, in Modern Aspects of Small-Angle Scattering, edited by $\mathrm{H}$. Brumberger, NATO ASI Series C: Mathematical and Physical Sciences, No. 451. Dordrecht: Kluwer.

Goerigk, G., Haubold, H.-G., Lyon, O. \& Simon, J.-P. (2003). J. Appl. Cryst. 36, 425-429.

Goodisman, J. \& Brumberger, H. (1971). J. Appl. Cryst. 4, 347351.

Haubold, H.-G., Gebhardt, R., Buth, G. \& Goerigk, G. (1994). Resonant Anomalous X-ray Scattering, edited by G. Materlik, C. J. Sparks \& K. Fischer, pp. 295-304. Oxford: Elsevier Science.

Haubold, H.-G., Hiller, P., Jungbluth, H. \& Vad, T. (1999). Jpn J. Appl. Phys. 38(Supp. 1), 36-39.

Haubold, H.-G., Wang, X. H., Goerigk, G. \& Schilling, W. (1997). J. Appl. Cryst. 30, 653-658.
Haubold, H.-G., Wang, X. H., Jungbluth, H., Goerigk, G. \& Schilling, W. (1996). J. Mol. Struct. 383, 283-289.

Haubold, H.-G., Vad, T., Waldoefner, N. \& Bönnemann, H. (2003). J. Appl. Cryst. 36, 617-620.

Hukins, D. W. L. (1981). X-ray Diffraction by Disordered and Ordered Systems. Oxford: Pergamon.

Jacoby, M. (2002). Chem. Eng. News, Aug. 5, pp. 26-32.

Li, Z.-H., Gong, Y.-J., Pu, M., Wu, D., Sun, Y.-H., Zhao, H. \& Dong, B.-Z. (2003). Wuji Huaxue Xuebao, 19, 252-256.

Meitzner, G. (1992). Characterization of Catalytic Materials, edited by I. E. Wachs, ch. 2. Stoneham, MA: Butterworth-Heineman.

Naudon, A. (1995). Anomalous Small-Angle X-ray Scattering, in Modern Aspects of Small-Angle Scattering, edited by H. Brumberger, NATO ASI Series C: Mathematical and Physical Sciences, No. 451. Dordrecht: Kluwer.

Polizzi (2002).

Ramaya, R. (1997). Study of Zeolite-Supported Platinum Catalysts Using Small Angle X-ray Scattering, $\mathrm{PhD}$ thesis, Syracuse University, USA.

Rasmussen, F. B., Molenbroek, A. M., Clausen, B. S. \& Feidenhans, R. (2000). J. Catal. 190, 205-208.

Schaefer, D. W., Brow, R. K., Olivier, B. J., Ricker, T. \& Beaucage, G. (1995). Characterization of Porosity in Ceramic Materials by SmallAngle Scattering: Vycor Glass and Silica Aerogel, in Modern Aspects of Small-Angle Scattering, edited by H. Brumberger, NATO ASI Series C: Mathematical and Physical Sciences, No. 451. Dordrecht: Kluwer.

Schmidt, P. W. (1995). Some Fundamental Concepts and Techniques useful in Small-Angle Scattering Studies of Disordered Solids, in Modern Aspects of Small-Angle Scattering, edited by H. Brumberger, NATO ASI Series C: Mathematical and Physical Sciences, No. 451. Dordrecht: Kluwer.

Somorjai, G. A., Powell, R. E., Montgomery, P. W. \& Jura, G. (1967). Small-Angle X-ray Study of Metallized Catalysts, in Small-Angle $X$-ray Scattering, Proceedings of the Conference Held at Syracuse University, edited by H. Brumberger. New York: Gordon and Breach.

Stiles, A. B. \& Koch, T. A. (1995).Catalyst Manufacture, 2nd ed. New York: Marcel Dekker.

Vad, T., Haubold, H.-G., Waldoefner, N. \& Bönnemann, H. (2002). J. Appl. Cryst. 35, 459-470.

Wachs, I. E. (1992). Editor. Characterization of Catalytic Materials. Stonehame, MA: Butterworth-Heineman. 